\section{Homo/bissexualidade masculina: um estudo sobre práticas sexuais desprotegidas em Fortaleza}

\section{Male homo/bisexuality: a study about unprotected sexual intercourse in Fortaleza, Brazil}

\section{Rogério C. Gondim}

Grupo de Apoio à Prevenção à AIDS - Ceará (GAPA-CE)

Rua Castro e Silva, 121/Sala 308 - Centro, 60.030-010 - Fortaleza/CE

Correspondência para/ Correspondence to:

Rua Júlio César, 420 - 60425-350 - Fortaleza/CE

Email: rgondim@brhs.com.br.

\section{Lígia R. S. Kerr-Pontes}

\section{Universidade Federal do Ceará}

Faculdade de Medicina - Departamento de Saúde Comunitária.

Rua Professor Costa Mendes, 1.608/5ªndar - 60.970-140 - Fortaleza/CE

Email: ligia@ufc.br

Trabalho baseado na tese intitulada - Sexo entre homens: um estudo sobre práticas sexuais e risco para o HIV em Fortaleza 1998, apresentada ao Departamento de Saúde Comunitária da Universidade Federal do Ceará, Faculdade de Medicina, em 30/10/1998.

Trabalho subvencionado pela Coordenação Nacional de DST/AIDS do Ministério da Saúde (Processo no. 081/06/96; Projeto no. 023/94)

\section{Resumo}

Estudo transversal realizado junto a 400 homens de prática homo/bissexual onde se buscou analisar fatores relacionados ao envolvimento em relações sexuais desprotegidas, entre homo/bissexuais masculinos de Fortaleza (CE), no período de maio a agosto de 1995. Os dados foram coletados através de questionário semi-estruturado, aplicado por 10 entrevistadores. Os entrevistados foram agrupados em 5 classes sociais e 4 faixas etárias. Foi realizada uma análise univariada entre a variável dependente (envolvimento com Relações Sexuais Desprotegidas - RSD) e os fatores predisponentes a estas, através de teste exato de Fischer. Dentre estes fatores, aqueles que se mostraram significativos foram incluídos na análise multivariada, através de regressão logística. Quarenta e sete por cento dos entrevistados se envolveram com relações sexuais desprotegidas (RSD) e os fatores relacionados a este envolvimento foram: não possuir informações básicas sobre transmissão do HIV/AIDS, ter tido uma freqüência de relação sexual com outro homem maior ou igual a 1 vez no mês nos últimos 12 meses, ter tido 1 ou mais contatos sexuais com mulheres nos últimos 12 meses, sentir se muito excitado com sexo desprotegido, mostrar atitudes negativas em relação ao Sexo Mais Seguro, não conhecer alguém com AIDS e a não participação em organizações homossexuais. Um contingente ainda grande de homens com prática homo/bissexual se envolve em práticas de risco, necessitando de um aumento do nível de informação, erotização de outras práticas de menor risco e o fortalecimento das relações sociais visando efetivar os programas de prevenção do HIV/AIDS junto a esta população.

Palavras-chave: Síndrome de imunodeficiência adquirida. HIV. Homossexualidade masculina. Fatores de risco. Comportamento sexual. 


\section{Abstract}

A cross-sectional study was carried out with 400 homo/bisexual men. Data were collected through a semi-structured questionnaire, applied by 10 interviewers. The objective was to analyze risk factors in the engagement of unprotected sexual intercourse (USI) among homo/bisexual men in Fortaleza (CE-Brazil). Interviewees were classified into 5 social classes and 4 age groups. A bivariate analysis between the outcome variable (involvement with unprotected sexual intercourse - USI) and the predictor variable was performed by means of Fischer's exact test. The predictor variables that were significantly related to the outcome variable in the bivariate analysis were included into a logistic regression model to assess their independent effects. Fortyseven percent of the men reported unprotected sexual intercourse. The predictor variables found to be significantly related to this involvement in the multivariate analysis were: less information on HIV transmission, having had sexual intercourse with another man once or more than once a month in the last 12 months, having had one or more than one sexual intercourse with women in the past 12 months, being sexually aroused with unprotected sex, showing negative attitudes towards safer sex, not knowing anybody with AIDS and not participating in homosexual organizations. This study suggests the need to improve the social network in order to attain an effective HIV/AIDS prevention program in this population.

Keywords: Acquired immunodeficiency syndrome. HIV. Homosexuality, male. Risk factors. Sex behavior.

\section{Introdução}

A epidemia da Síndrome da Imunodeficiência Adquirida (AIDS) é um dos mais graves problemas de Saúde Pública da virada do século.

Trata-se de uma epidemia rápida, feroz, com fortes repercussões sociais, cujo crescimento progressivo tem extrapolado as fronteiras das ciências biológicas, da saúde, tornando-se um problema que envolve todos os setores da sociedade e a dinâmica das relações interpessoais dentro das comunidades atingidas.

Estima-se que, na atualidade, existam 6 milhões de casos acumulados de AIDS, distribuídos mundialmente, com 24 milhões de pessoas infectadas pelo HIV, dos quais $58 \%$ são homens e $42 \%$ mulheres $^{1}$.

No Brasil, entre 1980 e 1996, a taxa de incidência acumulada de casos de AIDS passou de 0,005 por 100.000 habitantes para 63,1 por 100.000 habitantes, com um total de 88.099 casos notificados ao Ministério da Saúde. Destes, 52,9\% (46.575) ocorreram devido a exposição sexual ao HIV e dentro desta mesma categoria, $66,7 \%$ (31.074) foram devido a contatos homo/bissexuais masculinos $^{2}$.

Na região Nordeste, o estado do Ceará vem se destacando por apresentar uma concentração muita elevada de casos de AIDS entre homossexuais e bissexuais masculinos, embora também presencie modificações em seu perfil. Este traço da epidemia difere de outros estados na mesma região, onde um componente significativo de transmissões ocorre através do uso de drogas injetáveis.

O sexo masculino é responsável por $84,6 \%$ do total de casos registrados em todo o estado, no período de 1983 a 1996. Os casos de AIDS, ocorridos entre homossexuais ou bissexuais masculinos, representaram aproximadamente $53,5 \%$ deste total de ca$\operatorname{sos}^{3}$. No restante do Brasil, o percentual de casos nesta categoria vem decrescendo em relação às demais. Entre as categorias que vêm se destacando, assume um papel importante a transmissão heterossexual entre 
as mulheres que, neste estado, vem se processando principalmente através de contatos sexuais com parceiros bissexuais masculinos.

Estes dados nos mostram o quanto é significativa a transmissão do HIV através de contatos homo/bissexuais no Ceará, e que a prática sexual entre homens não é uma situação rara na região.

A prática do sexo anal seja insertivo, seja receptivo, sem o uso de preservativo de borracha (camisinha), é a prática sexual de maior risco para a transmissão do HIV ${ }^{4}$.

Neste sentido, os homens de práticas homo/bissexuais se colocam entre as populações de maior risco para a aquisição da infecção pelo HIV, em conseqüência da combinação da fragilidade e do alto poder de absorção da mucosa anal em contato com o sêmen, o principal veículo orgânico para o HIV.

O número de parceiros sexuais tem implicações sobre o risco de infecção pelo HIV, em especial entre os indivíduos com mais de 10 parceiros nos últimos 12 meses $^{5}$.

Mesmo considerando que, durante os primeiros 16 anos da epidemia de AIDS, houve várias mudanças nos comportamentos ou práticas sexuais que têm colocado estes homens sob o risco de HIV/AIDS, ainda persiste um considerável percentual de comportamento de risco ${ }^{6}$.

No Brasil, recente estudo realizado por Queiroz ${ }^{7}$, entre homo/bissexuais masculinos, concluiu que 51,9\% (135) não usavam preservativo sistematicamente. Os homens homossexuais tendem a se envolver mais em práticas sexuais de risco com parceiros regulares do que com parceiros casuais ${ }^{8}$. Outrossim, as práticas de relação anal sem preservativo são observadas, na sua grande maioria, entre homens que desconhecem o status sorológico dos seus parceiros para o HIV.

Assim, observa-se que os homens de prática homossexual, a despeito de qualquer mudança comportamental que possa ser observada, continuam se envolvendo em práticas (ou situações) de risco para a aquisição do HIV, em especial, relações anais sem o uso de preservativos, principalmente com seus parceiros regulares.

\section{Material e Método}

Trata-se de um estudo transversal, no qual foram aplicados 400 questionários semi-estruturados, no período compreendido entre maio e agosto de 1995, entre homens que referiam fazer sexo com outros homens (independente de terem relações sexuais com mulheres), residentes na região metropolitana de Fortaleza. O questionário utilizado foi uma adaptação local feita a partir de instrumentos semelhantes utilizados em outros estudos nacionais e internacionais $^{9,10}$.

Visando maximizar a representatividade do estudo, os indivíduos foram recrutados através de diferentes técnicas: recrutamento em áreas previamente identificadas sócio-culturalmente com atividades homossexuais, pelos pesquisadores, tais como, boates, bares, saunas e áreas de "pegação"* associadas à técnica denominada "bola de neve" ou redes de amizades (indicações de pessoas previamente entrevistadas). O processo de identificação destas áreas foi realizado por colaboradores que, em sua maioria, desenvolvem trabalhos preventivos nas áreas freqüentadas por homo/bissexuais masculinos e que pertencem a diferentes níveis sócioeconômico / culturais.

Todos os entrevistadores (em número de 10) eram homens de orientação homossexual. A opção por entrevistadores com as mesmas características da amostra visou facilitar a inserção dos mesmos junto ao grupo sob estudo e em ambientes gays onde seriam conduzidas as entrevistas. Os mesmos tendiam a pertencer às classes sociais $\mathrm{B}, \mathrm{C}$ ou D, fato este que pode ter colaborado para algum vício amostral. 
Visando, ainda, a maior representatividade possível da população estudada, uma vez que se trata de uma amostra de conveniência (não se conhece o universo da população de homo/bissexuais masculinos, seja em Fortaleza ou em outra área geográfica), procurou-se garantir que uma parcela dos entrevistados não fosse recrutada apenas através das redes de amizades.

Considerando-se que, tanto a classe social do indivíduo pesquisado, como a sua faixa etária poderiam ser fatores determinantes dos resultados encontrados, estabeleceu-se que o tamanho amostral deveria ser tal que garantisse no mínimo 10 indivíduos nos 20 grupos constituídos pela combinação das 5 classes sociais A (mais alta), B, C, D e E (mais baixa), baseadas no modelo de Guidi e Duarte ${ }^{11}$ que leva em consideração renda, ocupação e escolaridade de indivíduo, e das 4 faixas etárias $(<22,22$ । 32,32 - 42 e >42).

A divisão dos entrevistados nestas faixas etárias baseou-se na discussão realizada por Parquer ${ }^{10}$ sobre a interferência dos diferentes momentos históricos e contextos socioculturais vivenciados pelo país no comportamento dos homo/bissexuais masculinos.

Durante a análise dos resultados, as 5 classes sociais iniciais foram reagrupadas em 3 classes (A, B e C), de acordo com a descrição: "A" " agrupamento das classes iniciais A e B; "B"» classe inicial C e "C" " agrupamento das classes iniciais D e E.

Aos sujeitos do estudo foi garantido o sigilo das informações e o anonimato, de acordo com as normas éticas da investigação científica.

Devido às limitações impostas pelo próprio modelo do estudo (exposição e desfecho são levantados simultaneamente, fornecendo uma informação fotográfica de uma dada população em um dado período de tempo) e pelas características da população estudada (amostra de conveniência), não se podem generalizar os resultados deste estudo para toda a população de homens que fazem sexo com homens, assim como, para outros períodos de tempo, além de 1995.
Não obstante, os resultados aqui apresentados poderão servir como base/indicação das necessidades de saúde desta população, no tocante à prevenção do HIV/AIDS.

Uma análise univariada foi realizada entre a variável de desfecho (envolvimento com relações sexuais desprotegidas - RSD) e os fatores predisponentes a esta, através do teste exato de Fischer. Os fatores predisponentes que mostraram associação com a variável desfecho $(\mathrm{p}<0,15)$, na análise anteriormente referida, foram incluídos em uma análise logística multivariada para avaliar os efeitos independentes. Todas as possíveis variáveis preditoras foram analisadas univariadamente, sendo que a maioria apresentou-se significativa neste momento. Após análise por regressão logística muitas deixaram de ser significantes e não mostraram efeitos confundidos. Permaneceram no modelo somente as apresentadas nos resultados.

Visando evitar colinearidade entre as variáveis "escolaridade" e "nível de informação sobre HIV/AIDS”, a primeira foi excluída do modelo final de regressão. Nestas análises utilizou-se o software STATA(tm), versão 5.0, desenvolvido pela STATA Corporation, Texas, USA.

\section{Definição de variáveis}

\section{Envolvimento com relações sexuais} desprotegidas: (a) SIM - aqueles que responderam "sim" às seguintes práticas sexuais: foi penetrado no ânus sem camisinha ou penetrou o ânus de alguém sem camisinha ou fez sexo vaginal sem camisinha (nos últimos 6 meses anteriores a entrevista); (b) NÃO - aqueles que responderam "sim" ou "não" às seguintes práticas sexuais: foi penetrado no ânus com camisinha e penetrou o ânus de alguém com camisinha e fez sexo vaginal com camisinha (nos últimos 6 meses anteriores a entrevista).

Atitudes em relação ao sexo seguro: (a) POSITIVAS - são os entrevistados que responderam "concordo completamente" ou "em parte" com as seguintes questões: é fácil falar com um parceiro sexual que não 
vai transar sem usar preservativo; e discordam completamente ou em parte com: é difícil, no auge da excitação, fazer sexo mais seguro. (b) NEGATIVAS - são os entrevistados que "discordaram completamente" ou "em parte" com: é fácil falar com um parceiro sexual que não vai transar sem usar preservativo; e concordaram completamente ou em parte com: é difícil, no auge da excitação, fazer sexo mais seguro.

Sentem-se muito excitados com sexo desprotegido: SIM - são os entrevistados que responderam que se sentiam "muito excitado" ou "um pouco excitado" para pelo menos uma das seguintes questões: (a) tendo o ânus penetrado por um parceiro sem o uso de camisinha; (b) penetrando o ânus do parceiro sem camisinha; (c) tendo sexo vaginal sem camisinha. As respostas diferentes destas foram consideradas como NÃO.

\section{Resultados}

As características sociodemográficas dos participantes encontram-se descritas na Tabela 1.

Cerca de $70 \%$ dos participantes identificaram-se como homossexual/entendido/ gay, $16 \%$ identificaram-se como bissexuais. Quanto ao comportamento sexual, $72 \%$ dos entrevistados referiram ter tido sexo só com homens, nos últimos 12 meses anteriores á entrevista, porém, cerca de $23 \%$ tiveram sexo com homens e, em algum momento, sexo com mulheres.

O principal padrão de relacionamento sexual dos entrevistados, nos últimos 30 dias anteriores à entrevista, foi aquele de relações esporádicas com diferentes homens (35\%). Entre os que possuíam uma relação fixa com um homem (174), 44\% referiram este relacionamento há mais de 12 meses.

Dentre os entrevistados que relataram suas práticas sexuais, $47 \%$ se envolveram com relações sexuais desprotegidas (RSD) (sexo anal insertivo/receptivo sem camisinha ou sexo vaginal sem camisinha), nos últimos 6 meses anteriores à entrevista.
Na análise univariada, vários fatores mostraram-se associados significativamente com o envolvimento em RSD (Tabela 2). A proporção de homens mais jovens envolvidos em RSD foi significativamente maior do que os mais velhos ( $\mathrm{p}=0,017$ ). Os entrevistados que possuíam até o $1^{\circ}$ grau envolveram em RSD mais freqüentemente do que os que possuíam $2^{\circ}$ grau ou superior ( $\mathrm{p}=0,003)$. Não ser solteiro mostrou estar altamente associado com o envolvimento em RSD em relação a ser solteiro ( $\mathrm{p}=0,005)$.

Os indivíduos informados/mais ou menos informados sobre HIV/AIDS envolveram-se em RSD em menor proporção que aqueles não informados $(\mathrm{p}<0,001)$. Entretanto, mesmo entre os informados, $35 \%$ ainda se envolveram em RSD.

Os entrevistados que possuíam uma relação fixa com um homem nos últimos 30 dias anteriores à entrevista estavam mais propensos a se envolver em RSD do que aqueles que não possuíam tal relacionamento $(\mathrm{p}=0,005)$.

Os homens com mais de 10 parceiros nos últimos 12 meses se envolveram mais freqüentemente em RSD que aqueles com menos parceiros ( $\mathrm{p}=0,032)$. Também se encontrou associação entre os homens que tiveram uma maior freqüência de relações sexuais com homens nos últimos 12 meses e a prática de $\mathrm{RSD}(\mathrm{p}<0,001)$. Os entrevistados que tiveram relações sexuais com mulheres nos últimos 12 meses também se envolveram mais freqüentemente em RSD que aqueles que não o fizeram ( $\mathrm{p}=0,001)$.

As variáveis relacionadas com as atitudes dos entrevistados mostraram-se como fatores extremamente associados no envolvimento em RSD. Os indivíduos que se sentiam muito excitados com sexo desprotegido estavam muito mais propensos a se envolver em RSD do que aqueles que não referiram esta sensação $(\mathrm{p}<0,001)$. Da mesma forma, os que acreditavam ser o sexo anal a melhor maneira de fazer sexo $(68,6 \%)$ tendiam a se envolver mais freqüentemente em RSD do que aqueles que não pensavam assim $(p<0,001)$. Os entrevistados que apre- 
Tabela 1 - Distribuição dos entrevistados segundo as características sócio-demográficas.

Fortaleza, 1995.

Table 1 - Socio-demographics characteristics of the homo/bisexual men. Fortaleza, 1995.

\begin{tabular}{|c|c|c|}
\hline Características & Freq $^{*}$ & $\%$ \\
\hline \multicolumn{3}{|l|}{ Faixa etária } \\
\hline$\cdot<22$ anos & 76 & 19,0 \\
\hline$\cdot 22 \mid-32$ anos & 214 & 53,5 \\
\hline$\cdot>=32$ anos & 110 & 27,5 \\
\hline - Total & 400 & 100,0 \\
\hline \multicolumn{3}{|l|}{ Escolaridade } \\
\hline - $1 \circ$ grau inc. & 56 & 15,0 \\
\hline - $1^{\circ}$ grau com. & 27 & 7,2 \\
\hline - $2^{\circ}$ grau inc. & 41 & 10,9 \\
\hline - $2^{\circ}$ grau com. & 140 & 37,5 \\
\hline - Superior & 94 & 25,1 \\
\hline - Pós-graduado & 16 & 4,3 \\
\hline - Total & 374 & 100,0 \\
\hline \multicolumn{3}{|l|}{ Religião } \\
\hline - Afro-brasileira & 31 & 7,7 \\
\hline - Católica & 240 & 60,1 \\
\hline - Protestante & 14 & 3,5 \\
\hline - Espiritualista & 33 & 8,2 \\
\hline - Não tem religião & 67 & 16,7 \\
\hline - Outra & 15 & 3,7 \\
\hline - Total & 400 & 100,0 \\
\hline \multicolumn{3}{|l|}{ Estado civil } \\
\hline - Viúvo & 3 & 0,7 \\
\hline - Divorciado/desquitado/separado & 18 & 4,5 \\
\hline - Casado/junto/amigado & 27 & 6,8 \\
\hline - Solteiro & 352 & 88,0 \\
\hline - Total & 400 & 100,0 \\
\hline \multicolumn{3}{|l|}{ Classe social } \\
\hline - Classe A & 106 & 26,5 \\
\hline - Classe B & 170 & 42,8 \\
\hline - Classe C & 123 & 30,8 \\
\hline - Total & 400 & 100,0 \\
\hline \multicolumn{3}{|l|}{ Situação de moradia } \\
\hline - Com a mulher/família & 13 & 3,4 \\
\hline - Com o parceiro & 33 & 8,7 \\
\hline - Com amigos(as) & 55 & 14,5 \\
\hline - Sozinho & 56 & 14,7 \\
\hline - Com os pais/parentes & 223 & 58,7 \\
\hline - Total & 380 & 100,0 \\
\hline
\end{tabular}

*O total pode variar devido a valores ignorados

*The total may vary due unidentified values

sentaram atitudes negativas em relação ao sexo seguro são mais prováveis de se engajar em RSD do que aqueles que não apresentaram $(\mathrm{p}<0,001)$.
Não conhecer alguém com HIV/AIDS mostrou-se extremamente associado ao envolvimento em RSD em comparação àqueles que conhecem $(p<0,001)$. Da mes- 
Tabela 2 - Características dos entrevistados segundo o envolvimento em Relações Sexuais Desprotegidas (RSD). Fortaleza, 1995.

Table 2 - Characteristics of the homo/bisexual men according to the engagement in Unprotected Sexual Intercourse (USI), Fortaleza, 1995.

\begin{tabular}{|c|c|c|c|}
\hline Variáveis & $\mathrm{N}$ & RSD n(\%) & Valor de $\mathrm{p}^{*}$ \\
\hline \multicolumn{4}{|l|}{ Idade } \\
\hline$\cdot<22$ anos & 72 & $43(59,7)$ & 0,017 \\
\hline - 22 anos e mais & 275 & $120(43,6)$ & \\
\hline \multicolumn{4}{|l|}{ Educação } \\
\hline$\cdot 1^{\circ}$.grau & 70 & $42(60,0)$ & 0,003 \\
\hline - $2^{\circ}$. Grau ou superior & 252 & $98(38,9)$ & \\
\hline \multicolumn{4}{|l|}{ Estado civil - solteiro } \\
\hline - Sim & 304 & $134(44,1)$ & 0,005 \\
\hline • Não & 43 & $29(67,4)$ & \\
\hline \multicolumn{4}{|l|}{ Nível de informação sobre HIV/AIDS } \\
\hline - Informado/ Mais ou menos informado & 200 & $70(35,0)$ & $<0,001$ \\
\hline • Não informado & 147 & $93(63,3)$ & \\
\hline \multicolumn{4}{|l|}{ Relação fixa com um homem ( 30 dias) } \\
\hline - Sim & 146 & $82(56,2)$ & 0,005 \\
\hline • Não & 200 & $81(40,5)$ & \\
\hline \multicolumn{4}{|l|}{ No. Parceiros masculinos (12 meses) } \\
\hline - Menor/igual 10 parceiros & 227 & $97(42,7)$ & 0,032 \\
\hline - Maior 10 parceiros & 120 & $66(55,0)$ & \\
\hline \multicolumn{4}{|c|}{ Freqüência de relações sexuais com homens (12 meses) } \\
\hline - Menos 1 vez/mês & 153 & $49(32,0)$ & $<0,001$ \\
\hline - Mais ou igual a 1 vez/mês & 192 & $113(58,8)$ & \\
\hline \multicolumn{4}{|l|}{ Relações sexuais com mulheres ( 12 meses) } \\
\hline - Nenhuma & 266 & $111(41,7)$ & 0,001 \\
\hline - 1 ou mais & 81 & $52(64,2)$ & \\
\hline \multicolumn{4}{|l|}{ Sente-se muito excitado com sexo desprotegido } \\
\hline$\cdot \operatorname{Sim}$ & 288 & $155(53,8)$ & $<0,001$ \\
\hline • Não & 56 & $7(12,5)$ & \\
\hline - Negativas & 212 & $116(54,7)$ & \\
\hline \multicolumn{4}{|l|}{ Conhece alguém com AIDS } \\
\hline - Sim & 209 & $79(37,8)$ & $<0,001$ \\
\hline • Não & 125 & $77(61,6)$ & \\
\hline \multicolumn{4}{|l|}{ Envolve-se com organizações gays } \\
\hline - Sim & 36 & $8(22,2)$ & 0,002 \\
\hline • Não & 284 & $142(50,0)$ & \\
\hline
\end{tabular}

*Teste Exato de Fisher. O número total de entrevistados em cada categoria pode varia devido a valores ignorados. * The total number of interviewers can variate because of missing values.

ma forma, o não envolvimento com grupos homossexuais mostrou estar positivamente associado com a prática de RSD $(\mathrm{p}=0,002)$.

As variáveis que se mostraram significantes $(\mathrm{p}<0,15)$ na análise univariada, em relação ao envolvimento com RSD, foram analisadas para identificação de seus efei- tos independentes, em relação à RSD, através de um modelo de regressão logística (Tabela 3).

Os indivíduos não informados sobre HIV/AIDS foram mais propensos a se envolver em RSD. Os homens que tiveram 1 ou mais relações sexuais ao mês com outro homem, nos últimos 12 meses, e os que ti- 
Tabela 3 - Análise multivariada das variáveis relacionadas com o envolvimento em Relações Sexuais Desprotegidas (RSD) entre homo/bissexuais masculinos, Fortaleza, 1995.

Table 3 - Multivariate analyse of the variables related to the engagement in Unprotected Sexual Intercourse among homo/bisexual men, Fortaleza, 1995.

\begin{tabular}{lcc}
\hline Variáveis & $\begin{array}{c}\text { Odds Ratio Bruto } \\
\left(I C^{*} 95 \%\right)\end{array}$ & $\begin{array}{c}\text { Odds Ratio Ajustado** } \\
(\text { IC } 95 \%)\end{array}$ \\
\hline $\begin{array}{l}\text { - Não é informado sobre HIV/AIDS } \\
\text { - Teve freqüência de } 1 \text { ou mais relações } \\
\quad \text { sexuais ao mês com homens, nos últimos }\end{array}$ & $3,20(2,05-4,98)$ & $2,50(1,44-4,31)$ \\
$\begin{array}{l}12 \text { meses. } \\
\text { - Teve } 1 \text { ou mais relações sexuais com }\end{array}$ & $3,04(1,94-4,73)$ & $3,12(1,78-5,47)$ \\
$\quad$ mulheres, nos últimos 12 meses & $2,50(1,49-4,19)$ & $2,27(1,20-4,28)$ \\
- Sente-se muito excitado com sexo & & \\
$\quad$ desprotegido & $8,16(3,57-18,62)$ & $5,56(2,22-13,88)$ \\
- Apresenta atitudes negativas em relação & & \\
$\quad \begin{array}{l}\text { ao sexo seguro } \\
\text { - Não conhece alguém com AIDS }\end{array}$ & $2,70(1,69-4,31)$ & $2,76(1,56-4,88)$ \\
- Não se envolve com organizações gays & $2,63(1,67-4,16)$ & $1,74(1,01-3,04)$ \\
\hline
\end{tabular}

*IC - Intervalo de confiança (Confidence Interval).

**OR - Ajustado para todas as variáveis da tabela (Adjusted to all the other variables).

veram uma ou mais relações sexuais com mulheres, no mesmo período, também se envolveram mais freqüentemente em RSD. Os entrevistados que se sentiam muito excitados com sexo desprotegido, e aqueles que relataram atitudes negativas em relação ao sexo seguro estiveram mais propensos a se engajar em RSD. Os homens que não conheciam alguém com AIDS e os que não se envolveram com organizações gays também se envolveram mais freqüentemente em RSD.

\section{Discussão}

O estudo demonstra uma variedade de práticas e comportamentos sexuais desprotegidos entre homo/bissexuais masculinos na região metropolitana de Fortaleza e que podem aumentar o risco de infecção pelo HIV.

A comparação da escolaridade do grupo estudado com os casos de AIDS notificados entre 1983 e 1995 mostra que o grupo estudado apresentou uma maior escolaridade em relação aos casos notificados. Considerandose que o nível educacional mais alto tende a proteger os homens de um comportamento de risco, uma amostra mais representativa dos HSH em Fortaleza tenderia a mostrar uma maior prevalência de comportamentos de risco entre a população geral de HSH.

Ao contrário de outros estudos, as características sócio-demográficas dos entrevistados não apresentaram associação com o envolvimento em RSD.

A informação sobre a infecção pelo HIV/ AIDS é o primeiro passo para os indivíduos se prevenirem da contaminação, mesmo sabendo que a informação sozinha não é suficiente para isto. Neste estudo, um importante número de homo/bissexuais masculino (43\%) não possuía as mínimas informações sobre a infecção pelo HIV, em especial seus mecanismos de transmissão. A falta de informação leva a um maior risco de envolvimento com RSD. Informações básicas como o sêmen transmitir o HIV ou não, foram respondidas erroneamente num elevado percentual entre os entrevistados. Este achado difere de outros estudos ${ }^{10,12}$ onde a maioria dos entrevistados tinha as informações básicas sobre o HIV/AIDS.

A grande maioria dos entrevistados teve freqüência de uma ou mais relações sexuais com um homem ao mês, no último ano 
e este fator mostrou-se significativamente associado com RSD. Este achado pode estar retratando a ocorrência de relacionamentos regulares. Vários estudos têm apontado a estreita relação entre parceiros fixos e o não uso de preservativos ${ }^{13}$.

Tais estudos têm demonstrado que o fato de parceiros fixos se envolverem em RSD está muito mais relacionado à "crença" de que são soronegativos do que de fato o são. O envolvimento emocional entre os parceiros fixos leva-os a crer que não estão sob risco de adquirir o HIV através de RSD com este parceiro. Relações sexuais desprotegidas não são necessariamente inseguras para o HIV, desde que ocorram numa relação mutuamente monogâmica entre dois parceiros soronegativos.

O estudo não avaliou o status sorológico dos entrevistados, desta forma, não se pode afirmar que tais contatos sexuais sejam realmente de risco ou não para a transmissão do HIV. Entretanto, é bem provável que entre os $56 \%$ dos homens que referiram relação fixa com um outro homem e envolvimento com RSD, haja sorodiscordantes, fato este, que os colocaria sob risco para o HIV.

Trinta e um por cento dos que referiram um relacionamento fixo com um homem, tiveram sexo também com outros homens, revelando uma parcela importante de indivíduos não monogâmicos.

Entre aqueles que relataram uma relação mutuamente monogâmica com um homem, nos últimos 30 dias, $73 \%$ tiveram mais de 2 parceiros no último ano, anterior a entrevista. Este achado demonstra a provável existência da chamada "monogamia serial” entre os entrevistados. Tal comportamento monogâmico ocorre em série com parceiros sexuais regulares onde se observa a ocorrência de RSD. Neste caso, por ter um único parceiro a cada dado período, o indivíduo acredita não correr risco para o HIV, mesmo praticando sexo desprotegido com os mesmos.

Estudo realizado por McLean e col. ${ }^{13}$ mostrou que $66 \%$ dos homo/bissexuais masculinos investigados não percebiam como risco o envolvimento em RSD com um parceiro regular, independente de conhecer ou não o sorostatus do parceiro. $\mathrm{O}$ mesmo estudo demonstrou que quanto mais emocionalmente envolvidos com o parceiro, menos percepção de risco das RSD estes indivíduos irão apresentar.

No presente estudo, quase a metade dos entrevistados nunca fez sorologia anti-HIV e, portanto, desconhece plenamente sua situação sorológica. Apesar disso, muitos dos respondentes possuem relações fixas com um outro homem e estão envolvidos em RSD.

Este achado também poderia significar uma freqüência alta de relações sexuais com parceiros diferentes. O número de parceiros no último ano, tem-se mostrado significativamente associado com RSD em outros estudos $^{9,15}$, em especial, aqueles com 10 ou mais parceiros no último ano. Sob esta ótica, pode-se supor que um indivíduo com uma freqüência de relação sexual igual a "uma ou mais" vezes ao mês terá, no mínimo, mais de 10 parceiros no ano.

$\mathrm{O}$ atual estudo identificou que, entre aqueles indivíduos que relataram uma freqüência de relação sexual maior ou igual a uma vez no mês, $39 \%$ tiveram mais de 10 parceiros no último ano. Sabe-se que o número de parceiros sexuais é um forte fator para a transmissão do HIV. Quando isto ocorre na presença de RSD, este risco fica ainda mais evidente, uma vez que aumenta a possibilidade de contato com um indivíduo infectado e/ou subtipos virais mais infecciosos ${ }^{13,14}$.

Lazzarin e col. ${ }^{15}$, em estudo sobre soropositividade para o HIV entre homo/ bissexuais masculinos, observou que aqueles indivíduos soroconvertidos apresentavam uma elevada freqüência de relações sexuais com homens, anteriormente à soroconversão.

Um número importante de homens referiu, no mínimo, um contato sexual com uma mulher no último ano, mesmo entre os entrevistados que se identificam claramente como homossexuais/gays. Estes indivíduos estavam ainda muito mais propensos a 
se engajar em RSD. Cerca de dois terços dos que referiram um ou mais contato sexual com uma mulher no último ano, se envolveram com RSD. Homens com práticas bissexuais percebem o sexo com mulheres como de menor risco, em comparação ao sexo desprotegido com outros homens, e por isso tendem a não usar preservativos com as mesmas ${ }^{16}$. Neste sentido, o sexo com mulheres pode figurar para estes homens como uma alternativa à necessidade de usar preservativo no sexo com outros homens, prática esta vista como mais arriscada.

Parker $^{10}$ também destaca esta menor percepção de risco no sexo com mulheres em comparação ao sexo com homens. Tal percepção pode ainda ser acentuada pelo medo vivenciado pelos homens de práticas bissexuais, de que o uso de preservativo possa gerar suspeitas junto a suas parceiras de possíveis envolvimentos com relações homossexuais. Muitas mulheres desconhecem o comportamento bissexual de seus parceiros e por isso não se percebem sob risco para o HIV e não exigem a prática do sexo protegido ${ }^{16}$.

Ressalta-se o fato de que estes indivíduos que estão se envolvendo em relações sexuais desprotegidas com mulheres, também o fazem com seus parceiros masculinos. Nesta situação, independente da orientação sexual e da percepção de menor risco em relação ao sexo com mulheres, estes indivíduos não usam preservativos nem com homens, nem com mulheres.

Outro agravante desta situação reside no fato de que a mulher não é vista normalmente como capaz de transmitir o HIV para os homens, conseqüentemente, homens que se envolvem em relações vaginais tendem a não usar preservativo nestas situações ${ }^{16}$. Além disto, as mulheres também estão em desvantagem na negociação do sexo seguro com seus parceiros, devido à sua posição de subordinação na relação com o sexo masculino e à confiança de que seus parceiros não tenham atividade sexual fora de relação ou falariam com elas sobre possíveis riscos de infecção pelo HIV ${ }^{16}$.

Os homens que fazem sexo com ho- mens e com mulheres não pensam que podem estar colocando suas parceiras em risco de adquirir o HIV. Na verdade, o que eles percebem é que eles não correm risco de contrair o HIV de suas parceiras ${ }^{16}$.

A maioria dos entrevistados relatou grande excitação com sexo anal desprotegido, atitudes negativas em relação ao uso do preservativo e sexo anal como a melhor maneira de fazer sexo. Os dois primeiros fatores mostraram importante associação com o envolvimento com RSD entre os respondentes.

A sexualidade masculina está fortemente centrada na área genital e na penetração. Desta forma, os homo/bissexuais masculinos têm na prática anal uma dimensão importante de sua identidade sexual, que está sendo ameaçada pela possibilidade de transmissão do HIV/AIDS ${ }^{10}$.

O uso de preservativo pode ser uma barreira para estes homens experimentarem o prazer do sexo anal, pois no cenário da intimidade, a satisfação dada pelo sexo anal é extremamente importante, sendo o preservativo visto como uma frustração a esta intimidade. Assim, o sexo anal no cenário das relações sexuais entre homo/bissexuais masculinos pode ter um destacado significado simbólico e emocional que deve ser considerado nas intervenções preventivas ${ }^{17}$.

Neste estudo, "não conhecer alguém com AIDS" e "não se envolver com organizações gays” mostraram-se positivamente associados com o envolvimento em RSD. Parker $^{10}$ tem observado a falta de uma (sub)cultura homossexual em torno da identidade gay no Brasil. Desta forma, a ausência de um senso comunitário no tocante às questões (homo) sexuais pode limitar o desenvolvimento de uma cultura de sexo seguro e de responsabilidade pessoal.

O risco da AIDS afeta não somente indivíduos isolados, mas membros de um mesmo grupo social ${ }^{18}$. Assim, a ausência de uma rede social em torno da homossexualidade pode ter implicações importantes na implementação de intervenções eficazes para a prevenção do HIV/AIDS junto à população de homossexuais masculinos. 
Os estímulos mais significativos para a mudança de comportamento são externos, vêm dos grupos sociais, dos colegas (pares) e dos parceiros sexuais ${ }^{19}$. A aprendizagem vem da observação de modelos comportamentais de outras pessoas. Os indivíduos que ajustam seus comportamentos sexuais aos de seus parceiros sexuais ou colegas, poderão ser recompensados pela sua aceitação no grupo e pela gratificação de dar prazer ao outro.

Por outro lado, enquanto alguns círculos sociais podem apoiar positivamente a mudança de comportamento, outros já não o conseguem $^{20}$. É o que se pode observar no presente estudo, pois a maioria dos entrevistados se envolveu com algum ambiente social gay (bares, boites, saunas etc.). Entretanto, somente aqueles que estiveram envolvidos com organizações gays apresentaram um maior percentual de uso do preservativo.
As redes (relações) sociais dos homo/ bissexuais masculinos devem agir de forma a promover a mudança de normas sobre sexo desprotegido (ou protegido), favorecer a pressão dos colegas em relação à prática do sexo mais seguro e prover tais indivíduos de apoio social que reforce a continuidade da prática do sexo mais seguro e validar a orientação sexual de seus membros, suas identidades.

É necessário que os homo/bissexuais masculinos percebam a disponibilidade de apoio nas relações sociais, o que levará à redução dos sintomas de depressão e ansiedade e, conseqüentemente, à redução de práticas de risco para o $\mathrm{HIV}^{18}$.

Estudos futuros devem aprofundar tais questões, em especial, o papel das diversas relações sociais dentro da população de HSH em Fortaleza e suas implicações para a prevenção do HIV/AIDS.

\section{Summary}

A cross-sectional study was carried out with 400 homo/bisexual men in order to analyze risk factors in the engagement of unprotected sexual intercourse (USI) among these men in Fortaleza (CE-Brazil). Data were collected through a semi-structured questionnaire that was applied by 10 interviewers. Interviewees were classified into 5 social classes and 4 age groups. A bivariate analysis between the outcome variable (unprotected sexual intercourse - USI) and the predictor variables was performed by means of Fischer's exact test. The predictor variables that were significantly related to the outcome variable in the bivariate analysis were included into a logistic regression model to assess their independent effects. The mean age of the participants was 28.4 years. Thirty seven percent of these men had finished at least high school and the majority was catholic (60\%) and single (88\%). About $12 \%$ of the men had or had had a stable female partner. Fortythree percent were middle class and 59\% lived with their family. The majority (70\%) was self-identified as gay and $16 \%$ as bisexual men. Forty-seven percent of the men reported unprotected sexual intercourse. The predictor variables found to be significantly related to USI in the multivariate analysis were: men who had less information on HIV transmission, those who had had sexual intercourse with another man once or more than once a month in the last 12 months, those who had had one or more than one sexual intercourse with women in the past 12 months, those who felt more sexually aroused with unprotected sex, those that showed negative attitudes toward safer sex, those who did not know anybody with AIDS and those who did not participate in homosexual organizations. The main sexual behavior in the past 30 days had been occasional sexual intercourse with different men and 23\% reported sexual contact with anonymous partners. This study suggests the need to improve the social network in order to attain an effective HIV/ AIDS prevention program in this population. 


\section{Referências}

1. Mertens T, Low-Beer D. HIV and AIDS: where the epidemic going? Bull World Health Organ 1996; 74: 121-9.

2. Ministério da Saúde. Secretaria de Políticas de Saúde. Coordenação Nacional de Doenças Sexualmente Transmissíveis e AIDS. Bol Epidemiol AIDS 1996; 3: 21-57.

3. Secretaria de Estado da Saúde do Ceará. Departamento de Epidemiologia. Inf Epidemiol Sér Doenças Transm/ AIDS 1997; (1): 1-5.

4. Mertens T, Piot P. Global aspects of human immunodeficiency virus epidemiology: general considerations. In: DeVita VT, Hellman S, Rosenberg S A, editors. AIDS: etiology, diagnosis, treatment and prevention. 4th ed. Philadelfia: Lippincott-Raven; 1997. p. 103-18.

5. Webster RD. Second wave of HIV infection washes ashore: south beach health survey of young gay men. In: Abstracts of the $11^{\text {th }}$ International Conference on AIDS; 1996 Jul 7-12; Vancouver; Canada. p. 127.

6. Cornelisse EPA. A compararison of risk factors for HIV transmission between two cohorts of gay men. In: Abstracts of the $11^{\text {th }}$ International Conference on AIDS; 1996 Jul 7-12; Vancouver; Canada. p. 334.

7. Queiroz LG. Analysis of established relation between self-attributed identity and risk practices in Belo Horizonte, MG - Brazil gay community. In: Abstracts of the $11^{\text {th }}$ International Conference on AIDS; 1996 Jul 7-12; Vancouver; Canada. p.506.

8. Bartos MR. Gay men in regular relationships and HIV risk. In: Abstracts of the $11^{\text {th }}$ International Conference on AIDS; 1996 Jul 7-12; Vancouver; Canada. p.384.

9. Page-Shafer K, Veugelers PJ, Moss AR, Strarhdee S, Kaldor JM, Van Griensven GJP. Sexual risk behavior and risk factors for HIV-1 seroconversion in homosexual men participating in the tricontinental seroconverter study, 1982-1994. Am J Epidemiol 1997; 146: 531-42.

10. Parker R. A Construção da solidariedade: AIDS, sexualidade e política no Brasil. Rio de Janeiro: RelumeDumará; 1994.
11. Guidi MLM, Duarte SG. Um esquema de caracterização sócio-econômico. Rev Bras Estud Pedag 1969; 52(115): 65-83.

12. Parker R, Bastos C, Galvão J, Pedrosa JS, organizadores. $A$ AIDS no Brasil (1982-1992). Rio de Janeiro: Relume Dumará; 1994.

13. McLean J, Boulton M, Brookes M, Lakhani D, Fitzpatrick R, Dawson J, McKechnie R et al. Regular partners and risky behaviour: why do gay men have unprotected intercourse? Aids Care 1994; 6: 331-41.

14. Royce RA, Seña A, Cates W Jr, Cohen MS. Sexual transmission of HIV. N Engl J Med 1997; 336: 1072-8.

15. Lazzarin A, Saracco A, Nicolosi A. Man-to-woman sexual transmission of the human immunodeficiency virus: risk factors related to sexual behavior, man's infectiousness, and woman's susceptibility. Arch Intern Med 1991; 151: 2411-6.

16. Boulton M, Evans ZS, Fitzpatrick R, Hart G. Bisexual men: women, safer sex and HIV transmission. In: Aggleton P, Hart G, Davies P, editors. AIDS responses, interventions, and care. London: Falmer Press;1991. p.65-78.

17. DeZwart O. Anal sex among gay men: the hiv perspective and beyond. In: Abstracts of the $11^{\text {th }}$ International Conference on AIDS; 1996 Jul 7-12; Vancouver; Canada. p. 386.

18. O’Brien K, Wortman CB, Kessler RC, Joseph JG. Social relationships of men at risk for AIDS. Soc Sci Med 1993; 36: 1161-7.

19. Cohen C. Changing to safer sex: personality, logic and habit. In: Aggleton P, Hart G, Davies P, editors. AIDS responses, interventions, and care. London: Falmer Press; 1991. p.19-42.

20. Fisher JD. Possible effects of reference group-based social influence on AIDS-risk behavior and AIDS prevention. Am Psychol 1988; 43: 914-20. 